\title{
Association of AID and MUM1 by Immunohistochemistry in Diffuse Large B-Cell Lymphoma
}

\author{
Mardiah Suci Hardianti ${ }^{1, *}$, Syahru Agung Setiawan ${ }^{1}$, Nungki Anggorowati ${ }^{2}$, Wiwiek Probowati ${ }^{3}$ \\ ${ }^{1}$ Division of Hematology and Medical Oncology, Department of Internal Medicine, Faculty of Medicine, Public Health and Nursing, \\ Universitas Gadjah Mada/Dr. Sardjito General Hospital, Jl. Farmako, Yogyakarta, Indonesia \\ ${ }^{2}$ Department of Anatomical Pathology, Faculty of Medicine, Public Health and Nursing, Universitas Gadjah Mada/Dr. Sardjito General Hospital, \\ J1. Farmako, Yogyakarta, Indonesia
}

${ }^{3}$ Department of Internal Medicine, Universitas Kristen Duta Wacana/Bethesda Hospital, Jl. Dr. Wahidin Sudirohusodo No.5-25, Yogyakarta, Indonesia

${ }^{*}$ Corresponding author. E-mail: mardiah.suci@ugm.ac.id

Received date: Oct 14, 2020; Revised date: Nov 30, 2020; Accepted date: Dec 2, 2020

\section{Abstract}

B ACKGROUND: Diffuse large B-cell lymphoma (DLBCL) is a heterogeneous disease with dysregulation of somatic hypermutation (SHM) and class switch recombination (CSR) have been known to contribute for its lymphomagenesis. Activation-induced cytidine deaminase (AID) enzyme plays a vital role for both processes. Multiple myeloma oncogene 1 (MUM1) is known to upregulate the AID expression in normal and pathological conditions. However, both AID and MUM1 expression association in DLBCL is still unexplored using immunohistochemistry method. We examined DLBCL samples and then retrospectively tested its correlation with clinical findings.

METHODS: A retrospective cohort study with 20 cases of DLBCL biopsy tissue with AID and MUM1 antibody was conducted. The samples were then classified into concordant $\left(\mathrm{AID}^{+} / \mathrm{MUM}^{+}\right.$or $\left.\mathrm{AID}^{-} / \mathrm{MUM}^{-}\right)$and discordant group $\left(\mathrm{AID}^{-} / \mathrm{MUM}^{+}\right)$. The clinicopathological comparison was performed to observe any association

\section{Introduction}

As the most frequent type of B-cell lymphoma, diffuse large B-cell lymphoma (DLBCL) comprises for 30\%-40\% of this type of non-Hodgkin lymphoma, even more than $60 \%$ in Indonesia. $(1,2)$ Since two decades ago, DLBCL has been known as a heterogeneous disease both molecularly and between immunohistochemistry expression and clinical findings.

RESULTS: Among 20 samples of DLBCL, concordant expression rate of AID and MUM1 was 80\% with kappa Cohen's of $0.578(p=0.004)$. A significant association was observed between AID and MUM1 expression with a prevalence ratio of 2.25 (95\% CI: $1.08-4.67 ; p=0.008$ ). Clinical characteristics were not significantly different between each group. Restricted mean survival time was shorter in the concordant group compared with the discordant group but statistically insignificant (21.16 vs. 22.5 months; $p=0.531$ ).

CONCLUSION: The result of this study showed the association between AID and MUM1 expression in DLBCL. However, whether the association may add further molecular heterogeneity of DLBCL is still to be confirmed by expanding the study.

KEYWORDS: AID, CSR, DLBCL, MUM1, SHM

Indones Biomed J. 2021; 13(1): 48-54 clinically with the existence of two distinct types of DLBCL, namely germinal center B-cell (GCB) and activated B-cell $(\mathrm{ABC})$ or non-GCB, using the gene expression profiling. (3) The basic construction of the classification originates with the different developmental stage of B-cell, and its disruption may explain the arising of DLBCL. $(1,3,4)$

Somatic mutations could be viewed as one explanation of cancer, in which such defect accumulation may later 
transform the normal characteristic of cells into malignant phenotypes.(5) It has been known that several mutations of DLBCL were associated with the mechanism of class switch recombination (CSR) and somatic hypermutation (SHM). Activation-induced cytidine deaminase (AID) which is expressed by the aicda gene is an important enzyme for both two physiological processes.(6) On the other hand, AID is also recognized as the mutator enzymes that contributes for lymphomagenesis of DLBCL.(1,7) Previously, Aicda gene is more classified as a dominant gene expressed in germinal center cells, but in DLBCL AID expression is known to be more prominent in the ABC than GCB.(8) Clinically, AID overexpression has lower overall survival and progression free survival (PFS) in addition to poor response of salvage therapy after relapse in DLBCL. $(9,10)$

A transcription factor, namely multiple myeloma oncogene 1 (MUM1) or interferon regulatory factor 4 (IRF4), has been recognized as the marker of B-cells differentiation into plasma cells and their expression has an important role in upregulating the expression of AID for initiation of CSR.(11,12) Immunohistochemical staining with MUM1 is a marker of non-germinal center (nonGC) type of DLBCL.(3) Moreover, MUM1 expression in DLBCL is reported to have an impact on DLBCL clinical outcomes. $(13,14)$

In previous studies there was a correlation between AID and MUM1 expression with known close interactions between them physiologically. $(10,11)$ However, the expression between AID and MUM1 in local DLBCL samples and their association with clinical presentation have not been studied further in Indonesia although the cases are known to be quite often in a number. Thus, we aimed to examine the expression between AID and MUM1 among our local DLBCL samples using immunohistochemstry method to observe the association among them and further correlation with the clinical findings.

\section{Methods}

This was a retrospective study involving 20 cohorts of de novo DLBCL samples, not otherwise specified (NOS), diagnosed from January 2014 to December 2016. The tissue blocks were obtained from the Department of Anatomical Pathology, Dr. Sardjito General Hospital, Yogyakarta, Indonesia. Clinical parameters of all patients were extracted from patient's medical record, including patient's age, Ann-Arbor stadium, extra nodal tumor involvement, Eastern Cooperative Oncology Group
(ECOG) performance status, lactate dehydrogenase (LDH) level, chemotherapy regiment, and survival status. This study had been approved by The Ethical Committee of Faculty of Medicine, Universitas Gadjah Mada (No. KE/ FK/0173/2020).

\section{Immunohistochemistry Analysis}

For immunostaining, 4- $\mu \mathrm{m}$ thick sections were cut from formalin-fixed paraffin-embedded (FFPE) tissue blocks and placed on electrostatic-charged, poly-L-lysine-coated slides (Biogear, Microscope Slide, Biogear Scientific, BioVentures, Inc., Coralville, Iowa, USA). Sections were dehydrated at $45^{\circ} \mathrm{C}$ overnight. All immunostaining procedures including deparaffinization were performed on Semi-automatic Intellipath FLX (Biocare Medical, Concord, Massachusetts, USA) with open kit. The antigen retrieval process was performed on Deckloaking Chamber from Biocare Medical. The counterstaining process with hematoxylin was performed under a semi-automatic slide stainer. After that, the dehydration process was achieved, followed by clearing with xylene, and finally the mounting process was finished to end the entire immunostaining process.

The following primary antibodies were used in this study: AID (Invitrogen, eBioscience, San Diego, California, USA) and MUM1 (dilution: 1/50, clone: MUM1p, Dako SA, Glostrup, Denmark). Reactive lymph nodes tissue samples were used as positive controls. Negative controls were treated with the same immunohistochemical method by omitting the primary antibody. The cut-off level for interpreting MUM1 as positive was $>30 \%$ tumor cell staining.(15) However, the optimum cut-off of AID will be determined using subsequent ROC analysis, which resulted for $1 \%$ as threshold.

For this study, we grouped DLBCL based on AID and MUM1 IHC expression into the concordant and discordant group. The concordant group consisted of $\mathrm{AID}^{+} / \mathrm{MUM}^{+}$ and $\mathrm{AID}^{-} / \mathrm{MUM}^{-}$, however, the discordant group was only existed in $\mathrm{AID}^{-} / \mathrm{MUM1}^{+}$.

\section{Statistical Analysis}

Comparative, correlation, and survival analyses were performed using Rstudio (Version 1.3.959; R Foundation for Statistical Computing, Vienna, Austria). Receiver operating characteristic (ROC) curve was performed to obtain optimum cut-off and Kaplan-Meier curve was graphed to perform time-to-event analysis. Statistical significance was determined when $p<0.05$. Overall survival is defined as time from diagnosis to death resulting from any cause. 


\section{Results}

From 20 cases of DLBCL involved in this study, most cases had good ECOG performance status (95\%) and limited stage $(70 \%)$. Only few patients had multiple extranodal site $(10 \%)$. Many patients did not perform LDH level examination in their initial visit with only a patient among the available LDH group $(n=4)$ was known having increased level of LDH (25\%). Most patients were treated with rituximab based regiment $(90 \%)$. The baseline characteristics of the subjects could be seen in Table 1.

Representative expression between AID and MUM1 was shown in Figure 1. A diverse degree of expression of AID was found in either dark or light zones of germinal centers of the tumor area. Moreover, AID was mainly collocated in the cytoplasm of the tumor, despite in some cases were also positively stained in the nucleus (Figure B1). MUM1 was mainly stained in the nuclear area, however,

Table 1. Baseline characteristics of DLBCL samples.

\begin{tabular}{|c|c|}
\hline Parameter & n (\%) \\
\hline \multicolumn{2}{|l|}{ Sex } \\
\hline Female & $10(50)$ \\
\hline Male & $10(50)$ \\
\hline Age $($ mean $\pm S D)$, years old & $59.9 \pm 9.2$ \\
\hline \multicolumn{2}{|l|}{ Age Group } \\
\hline$\leq 60$ years old & $11(55)$ \\
\hline$>60$ years old & $9(45)$ \\
\hline \multicolumn{2}{|l|}{ Performance Status } \\
\hline Good & $19(95)$ \\
\hline Poor & $1(5)$ \\
\hline \multicolumn{2}{|l|}{ Stage } \\
\hline Limited & $14(70)$ \\
\hline Advanced & $6(30)$ \\
\hline \multicolumn{2}{|l|}{ Extranodal Sites } \\
\hline $0-1$ site & $18(90)$ \\
\hline 2 sites & $2(10)$ \\
\hline \multicolumn{2}{|l|}{ LDH Level (n=4) } \\
\hline Normal & $3(75)$ \\
\hline Elevated & $1(25)$ \\
\hline \multicolumn{2}{|l|}{ Regiment Type } \\
\hline Non-Rituximab & $2(10)$ \\
\hline Rituximab based & $18(90)$ \\
\hline
\end{tabular}

some cases also presented with weak to moderately-stained of the cytoplasm (Figure A2; Figure B2).

IHC examination obtained a significant association between AID and MUM1 in our local DLBCL sample $(p=0.008)$. The percentage of expression among DLBCL samples was provided in Figure 2. All AID positive samples had concordant positive of MUM1 expression with AID expression sample was 2.25 times to have same expression status of MUM1 (PR: 2.25; 95\% CI: 1.08-4.67). Statistical significance of concordant expression between AID and MUM1 was presented in Table 2. Concordance rate expression occurred in $80 \%$ of sample with Cohen's kappa of $0.578(p=0.004)$.

Clinicopathological comparison between concordant positive, negative, or discordant expression of AID and MUM1 was presented in Table 3. It was evidenced that no statistical difference in terms of age, stage, extranodal involvement, performance status, or LDH level ( $p>0.05$ ). Survival analysis with Kaplan-Meier curve based on concordant expression status between AID and MUM1 among DLBCL sample was presented in Figure 3. Overall, no significant difference was found between the concordant and discordant expression, with the latter type had lower hazard ratio of 0.99 (95\% CI: $0.10-9.65 ; p=0.992)$ compared with the former. In addition, restricted mean survival time was also shorter in concordant group but no significant difference was observed (21.16 vs. 22.5 months; $p=0.531$ ).

\section{Discussion}

The clinical characteristic of DLBCL subjects involved in this study was dominated by patient with predicted favorable factors, for instance, patient with limited stage, good performance status, and limited extranodal involvement. In addition, the DLBCL subjects age were similar with previous epidemiology study of DLBCL in Indonesia which predominates with patients aged of 50-59 years old.(2) On the other hand, the age was relatively younger than American, but comparatively similar with Asian, particularly in China.(16) Most patients were also treated using rituximab (anti-CD20) containing regiment as the real data world of DLBCL was shown favorable outcome using this therapy.(17)

Using the previous explained cut-off of expression, our preliminary study observed a high concordance expression rate between AID and MUM1 among our DLBCL samples. Moreover, the relatively moderate degree of expression 

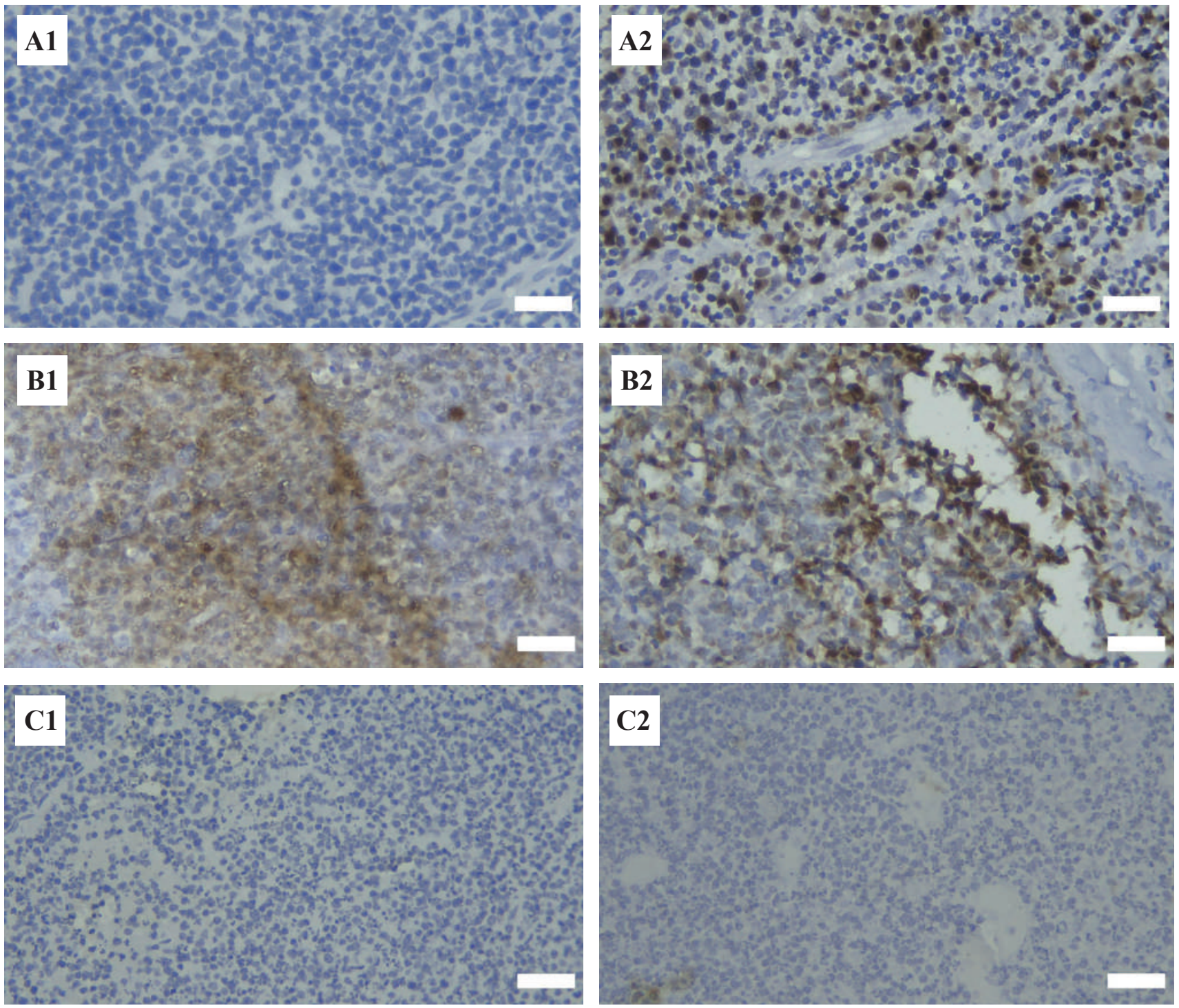

Figure 1. Representative IHC results of AID and MUM1 expression among DLBCL samples. A: Discordant (AID $\left./ \mathrm{MUM}^{+}\right)$; B: Concordant positive (AID $/ \mathrm{MUM1}^{+}$); $\mathrm{C}$ : Concordant negative (AID//MUM1 ${ }^{-)}$. White bar: $45 \mu \mathrm{m}$.

agreement observed by the kappa index obtained in this study may suggest the interplay between both genes expression in DLBCL. Similar association between AID and MUM1 expression was also observed by other study group.(10) Moreover, an earlier study also reported a predomination of non-GC or ABC to express AID along with MUM1, in which MUM1 was formerly recognized as a genetic marker of ABC DLBCL.(8) It has been known that MUM1/IRF4 was a transcription factor that may increase AID expression, directly or indirectly.(11,18) For translational relevance, this study may further confirm the diversity of DLBCL using the expression of AID and MUM1 which potentially add molecular information among DLBCL. The predomination of non-GC type of DLBCL in Indonesia also suggests an alternative measure to explain the diversity of this subtype using expression of AID and MUM1, as the latter gene expression was commonly found in this subtype.(19)

Clinicopathological comparison between the concordant and discordant expression of AID and MUM1 did not result any significant different clinical parameter for each group. The survival analysis also revealed insignificant survival differences in each group. Interestingly, the trend of a longer survival, even insignificant, in samples with AID negative but MUM1 positive, namely the discordant group, showed a tendency of favorable prognosis in DLBCL with AID negative. Molecularly, AID as a mutator enzyme may generate other oncogenic mutation such as IGH-MYC or IGH-BCL6 translocation, which later may transform 


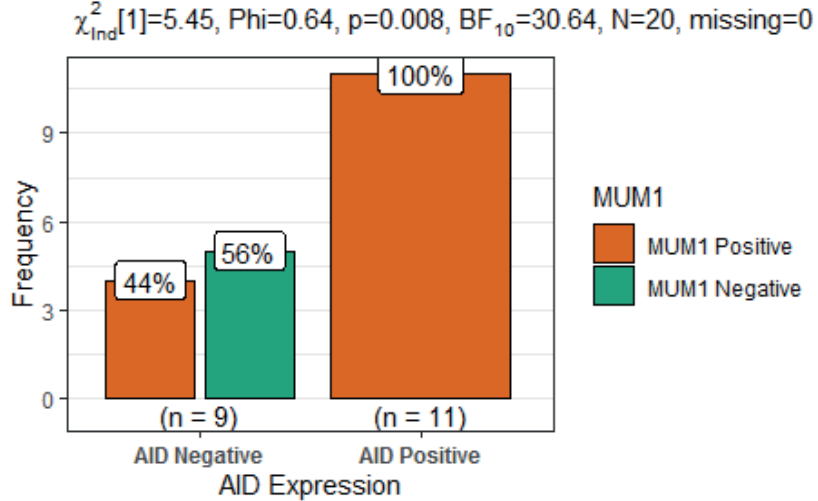

Figure 2. Frequency of AID and MUM1 expression among DLBCL samples.

into more aggressive type of lymphoma, namely molecular high-grade B-cell lymphoma. $(4,20,21)$ It was also proposed that expression of AID could indicate the alteration into more refractory type of lymphoma.(10,22)
Table 2. Association of concordant expression between AID and MUM1 among DLBCL samples.

\begin{tabular}{lc}
\hline \multicolumn{1}{c}{ Parameter } & Estimation \\
\hline Prevalence Ratio $(95 \% \mathrm{CI})$ & $2.25(1.08-4.67)$ \\
Concordance Expression $(95 \% \mathrm{CI})$ & $80 \%(58.4 \%-91.9 \%)$ \\
Cohen's Kappa (SE) & $0.578(0.17)$ \\
\hline
\end{tabular}

Thus, this preliminary study is still limited and needs to be expanded to examine any significant impact of expression status towards patient survival later.

Another limitation of the study was the discordant group only existed in sample with $\mathrm{AID}^{-} / \mathrm{MUM}^{+}$. Therefore, expanding the sample size in later research may increase the probability to find AID $^{+} /$MUM1 $^{-}$among DLBCL. The existence of this group also further confirms the characteristic distinction for each group and adds molecular heterogeneity of DLBCL.

Table 3. Clinicopathological comparison based on expression of AID and MUM1 among DLBCL samples.

\begin{tabular}{|c|c|c|c|c|}
\hline \multirow{3}{*}{ Paramater } & \multicolumn{3}{|c|}{ n (\%) } & \multirow{3}{*}{$p$-value } \\
\hline & \multicolumn{2}{|c|}{ Concordant } & \multirow{2}{*}{$\begin{array}{c}\text { Discordant } \\
{\text { AID } / \text { MUM1 }^{+}}^{+}\end{array}$} & \\
\hline & $\begin{array}{c}\text { Concordant Positive } \\
\left.\text { (AID }^{+} / \text {MUM1 }^{+}\right)\end{array}$ & $\begin{array}{c}\text { Concordant Negative } \\
\text { (AID/MUM1) }\end{array}$ & & \\
\hline $\mathrm{n}$ & 11 & 5 & 4 & \\
\hline Sex & & & & 0.27 \\
\hline Female & $4(40.0)$ & $4(40.0)$ & $2(20.0)$ & \\
\hline Male & $7(70.0)$ & $1(10.0)$ & $2(20.0)$ & \\
\hline Age $($ mean $\pm S D)$, years old & $62.00 \pm 8.59$ & $58.60 \pm 9.94)$ & $55.50 \pm 10.66)$ & 0.476 \\
\hline Age Group & & & & 0.576 \\
\hline$\leq 60$ years old & $5(45.5)$ & $3(27.3)$ & $3(27.3)$ & \\
\hline$>60$ years old & $6(66.7)$ & $2(22.2)$ & $1(11.1)$ & \\
\hline Performance Status & & & & 0.206 \\
\hline Good & $11(57.9)$ & $4(21.1)$ & $4(21.1)$ & \\
\hline Poor & $0(0.0)$ & $1(100.0)$ & $0(0.0)$ & \\
\hline Stage & & & & 0.21 \\
\hline Limited & $7(50.0)$ & $5(35.7)$ & $2(14.3)$ & \\
\hline Advanced & $4(66.7)$ & $0(0.0)$ & $2(33.3)$ & \\
\hline Extranodal Sites & & & & 0.457 \\
\hline $0-1$ site & $10(55.6)$ & $5(27.8)$ & $3(16.7)$ & \\
\hline 2 sites & $1(50.0)$ & $0(0.0)$ & $1(50.0)$ & \\
\hline LDH Level $(n=4)$ & & & & 1 \\
\hline Normal & $1(33.3)$ & $0(0.0)$ & $2(66.7)$ & \\
\hline Elevated & $1(100.0)$ & $0(0.0)$ & $0(0.0)$ & \\
\hline Regiment Type & & & & 0.403 \\
\hline Non-Rituximab & $2(100.0)$ & $0(0.0)$ & $0(0.0)$ & \\
\hline Rituximab based & $9(50.0)$ & $5(27.8)$ & $4(22.2)$ & \\
\hline
\end{tabular}



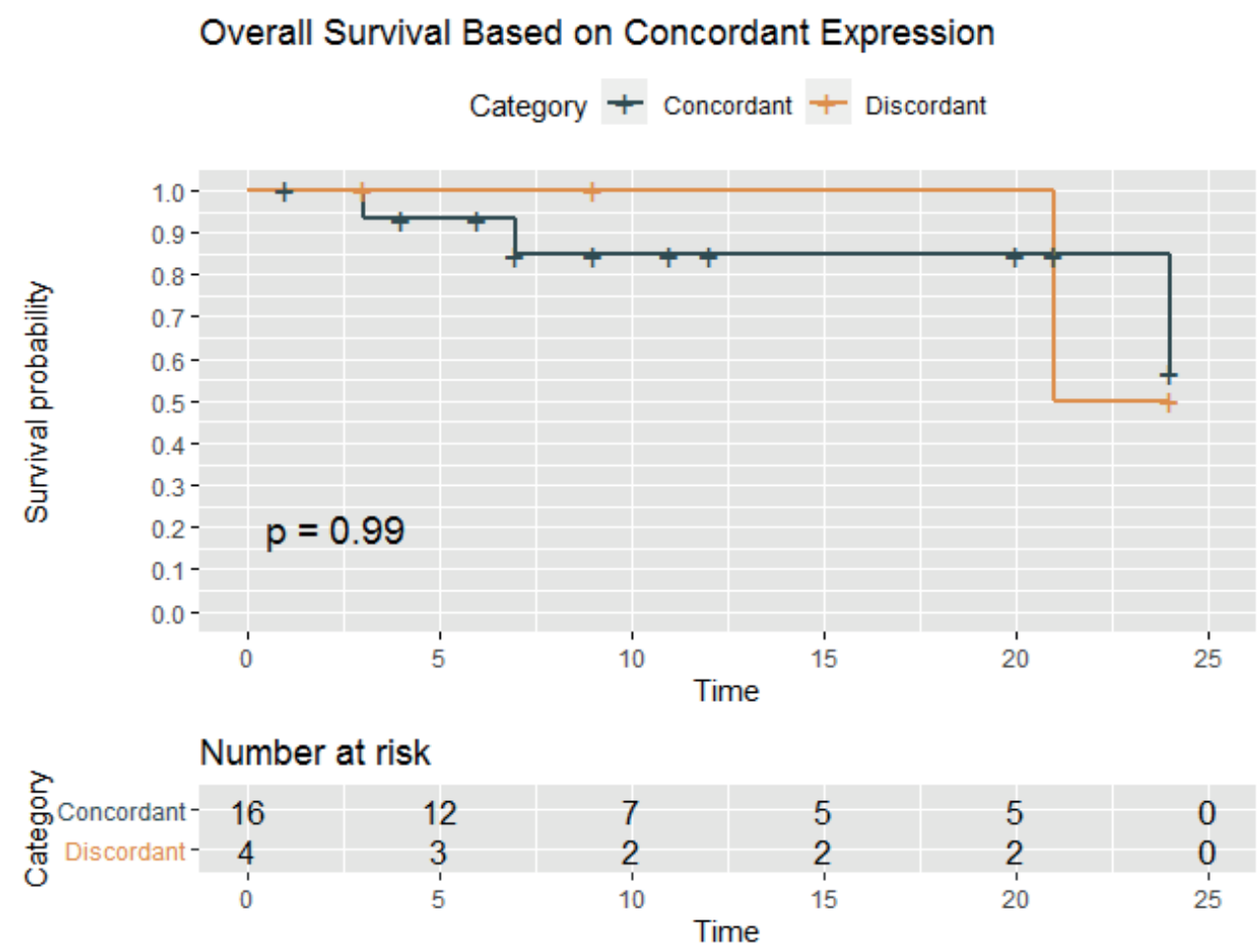

Figure 3. Overall survival in month-based on expression status among DLBCL samples.

\section{Conclusion}

In conclusion, our study confirmed the significant association of AID and MUM1 expression with immunohistochemistry methods to our local DLBCL samples. This association may potentially add molecular information of DLBCL type despite the insignificant differences in clinical presentation and survival, which is likely due to the limited samples. Expanding sample numbers and prolonging the evaluation period of study may further confirm any clinical impact of both AID and MUM1 expression in DLBCL.

\section{Acknowledgements}

The authors would like to thank for Agustina Supriyanti, Maria Reynelda Santoso, Ika Fi'ila Sari who assissted to perform immunohistochemical analysis of the samples, and also Macho Marcello, and Luthfia Kusuma Dewi who obtained the clinical data. Authors also acknowledge full support from PDUPT Grant of Indonesian Ministry of Research and Technology/National Agency for Research and Innovation 2020 (No. 2758/UN1.DITLIT/DIT-LIT/ PT/2020).

\section{Authors Contribution}

MSH concepted and designed the research. MSH, SAS, and NA performed the data acquisition/collection, while all authors were involved in the data analysis. MSH, NA, and WP interpreted the result. MSH and SAS prepared the manuscript and designed figures and tables. All authors took parts in giving critical revision of the manuscript and preparing proposal for the research funding.

\section{References}

1. Lenz G, Staudt LM. Aggressive lymphomas. N Engl J Med. 2010; 362: 1417-29.

2. Reksodiputro AH. Multicentre epidemiology and survival study of B cell non hodgkin lymphoma patients in Indonesia. J Blood Disord Transfus. 2015; 6: 2-6.

3. Sujobert P, Salles G, Bachy E. Molecular classification of diffuse large B-cell lymphoma. Hematol Oncol Clin North Am. 2016; 30: 1163-77.

4. Miao Y, Medeiros LJ, Li Y, Li J, Young KH. Genetic alterations and their clinical implications in DLBCL. Nat Rev Clin Oncol. 2019; 16: 634-52.

5. Meiliana A, Dewi NM, Wijaya A. Cancer stem cell hypothesis: Implication for cancer prevention and treatment. Indones Biomed J. 2016; 8: 21-36. 
6. Wang JH. The role of activation-induced deaminase in antibody diversification and genomic instability. Immunol Res. 2013; 55: 287-97.

7. Gu X, Shivarov V, Strout MP. The role of activation-induced cytidine deaminase in lymphomagenesis. Curr Opin Hematol. 2012; 19: 292-8.

8. Teater M, Dominguez PM, Redmond D, Chen Z, Ennishi D, Scott DW, et al. AICDA drives epigenetic heterogeneity and accelerates germinal center-derived lymphomagenesis. Nat Commun. 2018; 9: 222. doi: $10.1038 / \mathrm{s} 41467-017-02595-\mathrm{w}$.

9. Kawamura K, Wada A, Wang JY, Li Q, Ishii A, Tsujimura H, et al. Expression of activation-induced cytidine deaminase is associated with a poor prognosis of diffuse large B cell lymphoma patients treated with CHOP-based chemotherapy. J Cancer Res Clin Oncol. 2016; 142: 27-36.

10. Arima H, Fujimoto M, Nishikori M, Kitano T, Kishimoto W, Hishizawa $\mathrm{M}$, et al. Prognostic impact of activation-induced cytidine deaminase expression for patients with diffuse large B-cell lymphoma. Leuk Lymphoma. 2018; 59: 2085-95.

11. Hauser J, Grundström C, Kumar R, Grundström T. Regulated localization of an AID complex with E2A, PAX5 and IRF4 at the Igh locus. Mol Immunol. 2016; 80: 78-90.

12. Willis SN, Good-Jacobson KL, Curtis J, Light A, Tellier J, Shi W, et al. Transcription factor IRF4 regulates germinal center cell formation through a B cell-intrinsic mechanism. J Immunol. 2014; 192: 3200-6. doi: 10.4049/jimmunol.1303216.

13. Lu TX, Miao Y, Wu JZ, Gong QX, Liang JH, Wang Z, et al. The distinct clinical features and prognosis of the $\mathrm{CD} 10^{+} \mathrm{MUM1}^{+}$and CD10- Bc16- MUM1- diffuse large B-cell lymphoma. Sci Rep. 2016; 6: 20465. doi: 10.1038/srep20465.

14. Sofo-Hafizovic A, Chikha A, Gojak R, Hadzimesic A. Expression IRF/MUM1 $>25 \%$ predictor to three-year survival of diffuse large B cell lymphoma in the immunochemotherapy era. Med Arch. 2016; 70: $342-7$.
15. Coutinho R, Clear AJ, Owen A, Wilson A, Matthews J, Lee A, et al. Poor concordance among nine immunohistochemistry classifiers of cell-of-origin for diffuse large B-cell lymphoma: Implications for therapeutic strategies. Clin Cancer Res. 2013; 19: 6686-95. doi: 10.1158/1078-0432.CCR-13-1482.

16. Shi Y, Han Y, Yang J, Liu P, He X, Zhang C, et al. Clinical features and outcomes of diffuse large B-cell lymphoma based on nodal or extranodal primary sites of origin: Analysis of 1,085 WHO classified cases in a single institution in China. Chinese J Cancer Res. 2019; 31: 152-61.

17. Horvat M, Zadnik V, Južnič Šetina T, Boltežar L, Pahole Goličnik J, Novaković S, et al. Diffuse large B-cell lymphoma: 10 years' realworld clinical experience with rituximab plus cyclophosphamide, doxorubicin, vincristine and prednisolone. Oncol Lett. 2018; 15: 3602-9.

18. Ochiai K, Maienschein-Cline M, Simonetti G, Chen J, Rosenthal R, Brink R, et al. Transcriptional regulation of germinal center B and plasma cell fates by dynamical control of IRF4. Immunity. 2013; 38: 918-29.

19. Snak Y, Indrawati, Widayati K, Arfian N, Anggorowati N. Molecular subtypes, apoptosis and proliferation status in indonesian diffuse large B-cell lymphoma cases. Asian Pac J Cancer Prev. 2018; 19: 185-91.

20. Khodabakhshi AH, Morin RD, Fejes AP, Mungall AJ, Mungall KL, Bolger-Munro M, et al. Recurrent targets of aberrant somatic hypermutation in lymphoma. Oncotarget. 2012; 3: 1308-19.

21. Sha C, Barrans S, Cucco F, Bentley MA, Care MA, Cummin T, et al. Molecular high-grade B-cell lymphoma: Defining a poor-risk group that requires different approaches to therapy. J Clin Oncol. 2019; 37 : 202-12.

22. Zhang J, Shi Y, Zhao M, Hu H, Huang H. Activation-induced cytidine deaminase overexpression in double-hit lymphoma: potential target for novel anticancer therapy. Sci Rep. 2020; 10: 14164. doi: 10.1038/s41598-020-71058-y. 